\title{
Guía para la evaluación clínico-forense del daño psíquico consecutivo a los traumatismos craneoencefálicos.
}

\author{
Guide to forensic assessment of psychological impairment \\ after traumatic brain injury.
}

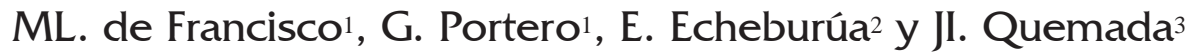

\section{RESUMEN}

En el ámbito de la Medicina Forense la evaluación clínica y funcional de las secuelas consecutivas a los traumatismos craneoencefálicos tiene importantes y trascendentes repercusiones en los distintos órdenes judiciales (penal, civil y laboral). Así, en el orden civil, la obligación de indemnización por daños origina la necesidad de Valoración del Daño Corporal. El juzgador establece la indemnización teniendo en cuenta la duración de los tiempos "legales" (de hospitalización, de curación o estabilización lesional en su caso y de impedimento), así como la presencia de secuelas y su cuantificación. La valoración del daño psíquico (neuropsicológico y neuropsiquiátrico) entraña una especial dificultad por cuanto en muchas ocasiones el daño no es fácilmente objetivable y cuantificable. Nuestro objetivo ha sido facilitar la evaluación clínico-forense de los traumatismos craneoencefálicos, protocolizando la evaluación y proponiendo una guía clínica y funcional de las secuelas neuropsicológicas y neuropsiquiátricas. Para ello se ha llevado a cabo una amplia revisión de los aspectos teóricos relativos a los traumatismos craneoencefálicos, que ha servido de base para la elaboración de la guía. En ésta se describen las principales secuelas y su forma de evaluación (clínica y mediante tests estandarizados en el caso de secuelas neuropsicológicas).

Palabras clave: daño cerebral traumático, Daño corporal, Evaluación neuropsicológica, Peritación médico forense, Secuelas psíquicas, Traumatismo craneoencefálico.
ABSTRACT

In Forensic Medicine, clinical and functional assessment of sequelae after traumatic brain injury has significant medico-legal implications. The duty to compensate for the damage caused leads to the "assessment of the organic and psychological harm inflicted". Judges establish financial compensation according to the duration of the "legal periods" (time of hospitalization, time till cure or time to lesion stabilization) and the quantification of the permanent squeals. Assessment of psychological impairment (neuropsychological and neuropsychiatric) is particularly difficult due to the lack of objective and quantifiable data. The aim of this work is to facilitate the forensic assessment by proposing a clinical and functional guide that helps to standardize the evaluation of neuropsychiatric and neuropsychological squeals. A wide review of the literature has been the basis for the proposed protocol. Neuropsychological tests as well as neuropsychiatric diagnostic tools are included.

Key words: traumatic brain injury, neuropsychological assessment, forensic evaluation, psychological sequels, traumatic brain injury.

Correspondencia: Dra. Mํㅡㄴ Luisa de Francisco Maíz. IVML, Subdirección de Vizcaya. Clínica Médico Forense. Calle Buenos Aires 6, $4^{a}$ planta. 48001 Bilbao. Teléfonos: 630030103 - 944016 484. Correo electrónico: francisco.ml@AJU.ej-gv.es.

\footnotetext{
${ }^{1}$ Médico Forense del Instituto Vasco de Medicina Legal (IVML), Subdirección de Vizcaya.

2 Catedrático de Psicología Clínica. Facultad de Psicología de la Universidad del País Vasco.

3 Servicio de Daño Cerebral. Hospital Aita Menni. Bilbao.
} 


\section{INTRODUCCIÓN:}

Los traumatismos craneoencefálicos (TCE) representan un grave problema en los países industrializados, no sólo por su elevada incidencia, sino también por afectar principalmente a un grupo de población joven. En ocasiones, sus secuelas resultan total o parcialmente invalidantes, lo cual acarrea graves repercusiones de tipo sociofamiliar y económico. La Organización Mundial de la Salud (OMS) considera a la lesión cerebral una de las causas de mayor coste social para el siglo XXI. De hecho, supone hoy en día el 2\% del PIB de cada país. En el ámbito clínico, el aumento en la eficacia de los tratamientos médicos intensivos ha permitido que los índices de mortalidad disminuyan, pero este hecho genera un incremento del número de personas afectadas por las diferentes secuelas derivadas de esta lesión cerebral. De entre las diferentes secuelas, las neuropsicológicas y neuropsiquiátricas plantean la máxima dificultad de peritación y estimación del daño.

La prevalencia de los trastornos neuropsicológicos y neuropsiquiátricos entre los afectados por un traumatismo craneoencefálico es muy elevada [I], dado que la incidencia del daño cerebral de origen traumático es de entre 100/300 cada 100.000 habitantes [2, 3]. La severidad del daño cerebral viene determinada por la naturaleza de las lesiones sufridas en el momento del impacto y por la aparición de complicaciones secundarias.

La etiología de los TCE está fuertemente asociada con la forma de vida en los países desarrollados: accidentes de tráfico, accidentes laborales, agresiones, caídas y deportes u ocio [4, 5].

El sistema jurídico demanda del Médico Forense asesoramiento en la valoración diagnóstica y de impacto funcional de múltiples tipos de patologías. Las lesiones cerebrales y sus consecuencias neuropsicológicas y neuropsiquiátricas se encuentran entre las de más difícil evaluación y de mayor trascendencia para la calidad de vida de los lesionados.

En el ámbito de la Medicina Forense la evaluación clínica y funcional de las secuelas neuropsicológicas y neuropsiquiátricas tiene importantes y trascendentes repercusiones en los distintos órdenes judiciales: penal, civil y laboral. Así, en el orden civil, la obligación de indemnización por daños origina la necesidad de Valoración del Daño Corporal. La Ley 34/2003, de 4 de noviembre, de modificación y adaptación a la normativa comunitaria de la legislación de seguros privados [6], establece un sistema de baremación para la indemnización de daños corporales.

La valoración del daño neuropsicológico y neuropsiquiátrico consecutivo a un traumatismo craneoencefálico entraña especial dificultad, por cuanto en muchas ocasiones el daño no es fácilmente objetivable y cuantificable.

El objetivo principal de este trabajo es facilitar la evaluación clínico-forense de los traumatismos craneoencefálicos, protocolizando la evaluación y proponiendo una guía para la evaluación clínica y funcional de las secuelas neuropsicológicas y neuropsiquiátricas.

Una parte de la valoración del daño corporal en general, y psíquico en particular, lo constituye la determinación de la duración de los "tiempos legales", a saber: tiempo de hospitalización, tiempo de impedimento y tiempo de curación. Estos tiempos son utilizados por los Jueces y Tribunales para la indemnización del daño corporal, por lo que de su resultado deriva en parte la cuantía económica de la indemnización. La otra parte, en lo que a la evaluación del daño corporal se refiere, se calcula teniendo en cuenta las secuelas resultantes del traumatismo craneoencefálico: físicas, neurológicas, neuropsicológicas y neuropsiquiátricas. Por tanto, en una evaluación clínica forense deben constar los principales elementos de juicio (tiempos legales y secuelas) para el resarcimiento del daño psíquico.

La inquietud por elaborar una guía para este tipo de valoraciones periciales está en el origen de esta investigación. La corrección de infravaloraciones del daño, la reducción de fraudes por 
exageraciones del mismo y la reducción de la litigiosidad, serían las consecuencias prácticas de la utilización de una guía de estas características.

La determinación de un pronóstico después de un traumatismo craneoencefálico constituye una cuestión fundamental, no sólo desde la perspectiva del clínico, sino desde la del propio paciente, de la familia y de la Administración. Por su parte, las entidades aseguradoras y los servicios responsables de cubrir los gastos que requieren la atención y los cuidados de estos pacientes también solicitan información sobre el pronóstico desde el periodo inmediatamente posterior al traumatismo. Ello influye en la toma de decisiones sobre la provisión de recursos y servicios a lo largo de todo el proceso rehabilitador, así como en la consignación de fondos económicos o aportaciones económicas previas a la resolución judicial, para evitar el pago de los intereses al tratarse de indemnizaciones cuantiosas.

Este trabajo parte de una amplia revisión de los aspectos teóricos de las secuelas consecutivas a los traumatismos craneoencefálicos. Se han analizado el concepto, la clasificación, los mecanismos de producción, la fisiopatología de los traumatismos craneoencefálicos, las lesiones cerebrales traumáticas, las secuelas de los traumatismos craneoencefálicos y su diagnóstico, la evaluación clínico-forense, la simulación y los aspectos médico-legales.

Con dicha información hemos elaborado una guía de evaluación clínico-forense que permita diagnosticar secuelas neuropsicológicas y neuropsiquiátricas. Asimismo se propone un sistema de evaluación de secuelas psíquicas que se corresponda con las clasificaciones internacionales de las enfermedades psíquicas: DSM-IV-TR [7] y CIE I0 [8]. Las categorías diagnósticas que se refieren a los trastornos psiquiátricos encontrados en pacientes que han sufrido una lesión cerebral traumática están presentes tanto en el DSM-IV-TR como en la ClE- I O, que presentan pequeñas diferencias terminológicas, pero no de fondo. En concreto, en este trabajo se ha optado por el DSM-IV-TR en la evaluación de secuelas psiquiátricas consecutivas a traumatismos craneoencefálicos.

\section{VALORACIÓN MÉDICO-FORENSE DEL DAÑO PSÍQUICO CONSECUTIVO A LOS TRAUMATISMOS CRANEOENCEFÁLICOS:}

En la valoración del daño corporal en general, y del consecutivo al TCE en particular, el análisis médico debe comprender los siguientes puntos fundamentales:

- Datos del explorado.

- Fecha del TCE.

- Etiología médico-legal del TCE.

- Antecedentes personales: somáticos, psíquicos, tóxicos y de traumatismos previos.

- Determinación del estado de salud o enfermedad del lesionado en el momento previo al accidente, es decir, el estado anterior (concausas previas).

- Severidad del TCE: Escala de coma de Glasgow, duración del coma y duración de la amnesia postraumática.

- Estudio de las lesiones provocadas por el TCE.

- Establecimiento del nexo de causalidad entre el TCE y las lesiones producidas.

- Evolución de las lesiones sufridas, así como de las incidencias acaecidas durante dicha evolución.

- Determinación de concausas concomitantes o posteriores al TCE que hayan podido incidir en su evolución.

- Tratamientos efectuados, incluyendo rehabilitación física, neuropsicológica y logopedia.

- Determinación de tiempos legales: duración de los periodos de hospitalización, 
incapacitación y de curación o de estabilización lesional en su caso.

- Determinación de secuelas resultantes, debiendo realizar una evaluación objetiva y exacta, en sus aspectos tanto anatómicos como funcionales.

- Establecimiento de las consecuencias de las secuelas neuropsicológicas y neuropsiquiátricas sobre las actividades que desarrollaba habitualmente el individuo, tanto en el ámbito laboral, como en el académico, social, personal, familiar, etc.

A continuación se comentan algunos de los elementos fundamentales para la valoración del daño psíquico. Dada la extensión del articulo y su objetivo, no se tratan en profundidad, teniendo en cuenta que cada uno de ellos podría ser objeto de una monografía.

\section{Relación de causalidad entre el traumatismo craneoencefálico, lesiones producidas y secuelas neuropsicológicas y neuropsiquiátricas.}

La valoración de los efectos de un TCE precisa la comprensión de la fisiopatología y la realización de estudios de neuroimagen, que aportan datos estructurales y funcionales relevantes [9, I0, I I]. Las alteraciones psíquicas en los TCE están directamente relacionadas con los mecanismos fisiopatológicos subyacentes y con variables biológicas y demográficas.

El estudio del nexo de causalidad (relación causa-efecto) en la valoración del daño neuropsicológico y neuropsiquiátrico es capital, ya que sin causalidad no ha lugar la compensación. Se deben considerar los elementos de juicio siguientes:

- Certeza del diagnóstico etiológico.

- Realidad e intensidad suficiente del TCE.

- Ausencia de patología anterior e integridad de la función.

- Concordancia anatómica y/o funcional entre el TCE y las secuelas neuropsicológicas y neuropsiquiátricas.

- Plazo transcurrido entre el TCE y la aparición de la lesión y/o secuela.

- Continuidad evolutiva o encadenamiento clínico.

- Certeza en el diagnóstico actual.

- Exclusión de otras hipotéticas causas.

Estos criterios han sido recogidos por muchos autores, como López Gómez y Gisbert Calabuig [12], y siguen principalmente los criterios etiológicos, topográficos, cronológicos, cuantitativos y de continuidad sintomática.

Para establecer la relación de causalidad entre el TCE y su resultado, debe atenderse a estos criterios, si bien hay que tener en cuenta las peculiaridades de los TCE: existencia de lesiones cerebrales por contragolpe, periodos de latencia dilatados en algunos hematomas subdurales, etc. Para el médico y/o perito esta relación se basa en datos etiopatogénicos.

Para estimar la probabilidad de etiología traumática en el origen de un cuadro psiquiátrico, Quemada et al. [13] señalan cinco criterios relevantes:

- Proximidad temporal. A mayor proximidad temporal entre la lesión cerebral y el debut del síndrome psiquiátrico, mayor fuerza cobra la relación causal, al menos como factor precipitante. Estos cuadros no suelen diagnosticarse mientras el paciente está en estado de confusión postraumática; tan pronto como esta última se resuelve los cambios de personalidad empiezan a hacerse evidentes. Este es sin duda el trastorno psiquiátrico más prevalente. Ello no implica que no se produzcan cambios en la expresión psicopatológica del trastorno, con la apatía, las conductas desinhibidas o la inestabilidad emocional resultando predominantes en momentos distintos. En el caso de los trastornos 
bipolares, la experiencia parece sugerir que debutan muy poco tiempo después de acabar este período (días, semanas); las psicosis esquizofreniformes, con una baja prevalencia, pueden debutar trascurridos varios meses.

- Patrón psicopatológico. La presencia de síntomas atípicos, tales como los trastornos de la identidad, son sugerentes de psicosis secundarias al TCE. También son sugerentes de psicosis secundarias a TCE aquellos casos en los que una alteración persistente de atención o memoria de lugar a una explicación de naturaleza delirante que venga a mitigar la angustia o el desconcierto provocados por la conciencia del déficit cognitivo.

- Déficit neuropsicológico asociado. La presencia de un déficit neuropsicológico asociado confirma la existencia de un TCE de intensidad suficiente como para provocar un tipo de alteración cognitiva no esperable en un paciente con esquizofrenia o trastorno bipolar.

- Neuroimagen positiva. La constatación de lesiones cerebrales en pruebas de neuroimagen estructural (TAC o RNM) introduce también un elemento diferenciador con respecto a los hallazgos habituales en enfermedades psiquiátricas.

- Antecedentes familiares. Aunque la presencia o ausencia de antecedentes familiares no constituye por sí solo argumento absoluto para defender una u otra etiología, la presencia de antecedentes familiares del mismo tipo de trastorno incrementa la probabilidad de que el paciente presentara una vulnerabilidad previa sobre la que el TCE haya actuado.

\section{Determinación de concausas.}

En ocasiones el resultado lesivo de una acción traumática no se debe únicamente al mecanismo lesional, sino a la concurrencia y convergencia de otros factores. A éstos se les llama concausas o factores extraños a los medios o procedimientos que son causa de lesiones o secuelas y que, sin embargo, se insertan en el proceso dinámico del que son consecuencia, de tal manera que el resultado último que se obtiene resulta diferente al que cabría esperar, dadas la naturaleza e intensidad del agente principal.

Existen tres tipos de concausas: preexistentes, concurrentes y consecutivas. Las concausas preexistentes se refieren al estado anterior del lesionado, estado que puede agravar los resultados del TCE. Las concausas concurrentes son aquellas que actúan de forma simultánea y aumentan la entidad del resultado final y, por último, las consecutivas serían las complicaciones, ajenas a la voluntad del lesionado, que pueden surgir durante el proceso de curación y evolución de las lesiones consecutivas a un TCE.

\section{Determinación de los tiempos legales.}

La determinación de la duración (en días) del periodo de hospitalización, impedimento y de curación o estabilización en su caso, sirve para determinar las indemnizaciones por lesiones permanentes e incapacidad temporal, que resultan de aplicar el sistema para la valoración de daños y perjuicios causados a las personas en accidentes de circulación.

El Ministerio de Economía y Hacienda actualiza anualmente las cuantías de las indemnizaciones. Las correspondientes al año 2008 se publicaron en el BOE núm 21, de 24 de enero de 2008 [ I 4]. La tabla V establece las indemnizaciones básicas por incapacidad temporal:

- Durante la estancia hospitalaria: 64,57 €/día

- Sin estancia hospitalaria:

- Impeditivo: 52,47 €/día

- No impeditivo: 28,26€/día 
La primera consecuencia que se deriva del TCE grave, desde el punto de vista asistencial, es la necesidad de ingreso hospitalario para la práctica de tratamientos intensivos y maniobras diagnósticas y terapéuticas que su situación requiera.

La segunda consecuencia es la imposibilidad de llevar a cabo sus actividades habituales (profesionales, deportivas, familiares, etc.).

La tercera es la necesidad de someterse a cuantos procedimientos terapéuticos se estimen necesarios para alcanzar la curación, o estabilización lesional.

Para la determinación del periodo de curación o estabilización lesional en su caso, es recomendable seguir los siguientes criterios médico-legales:

I.El lesionado puede reincorporarse a su trabajo habitual sin afectación para la lesión que causó la baja, o se establece claramente algún grado de invalidez permanente.

2.El lesionado no precisa más asistencia o controles médicos.

3. El lesionado ha alcanzado el máximo de restitución anatómica y funcional, encontrándose en el momento más cercano al estado prelesional.

La estabilización de los trastornos, desde el punto de vista médico-legal, se alcanza cuando la terapéutica activa curativa haya finalizado, ayudados por los tiempos de consolidación médico-legal habituales de la patología, establecidos por la bibliografía y la experiencia.

Obtenidos los "tiempos legales" (en días), éstos serán compensados económicamente según establezca la legislación al efecto o determine el arbitrio judicial.

\section{Determinación de las secuelas resultantes.}

La valoración de secuelas se plantea cuando la recuperación no ha alcanzado la completa restitución anatómica y/o funcional, quedando un daño permanente $[14,15,16]$. Este daño puede ser de tal entidad que provoque un estado de incapacidad que afecte a una o más esferas de la vida del lesionado.

Las secuelas neuropsicológicas y neuropsiquiátricas son el resultado final del proceso de curación cuando el mismo no ha sido "ad íntegrum". Son consecuencias evidentes del evento traumático y susceptibles de cuantificar, por lo que ya desde antiguo han sido la base de la valoración de los daños a las personas. Gracias a los avances de las técnicas diagnósticas, en los últimos decenios se han desarrollado numerosas y completas relaciones de secuelas con su correspondiente valor asignado, lo que constituye el elemento central de los actuales baremos y tablas de valoración. El perito debe determinar y concretar la deficiencia que la secuela produce, procurando ajustarse a los epígrafes de la ley sobre los baremos publicados en el BOE [17].

\section{Secuelas neuropsicológicas.}

Las secuelas neuropsicológicas no siempre son evaluadas adecuadamente, ya que son menos evidentes que las físicas y, por tanto, más difíciles de objetivar clínicamente.

Los objetivos fundamentales de la evaluación neuropsicológica se centran en identificar alteraciones de las funciones reguladas por la corteza cerebral, es decir, en el diagnóstico del deterioro que muestra el traumatizado y en la determinación de su organicidad.

En los últimos años se han encontrado correlaciones entre la topografía de la lesión cerebral con defectos neurológicos específicos y los cambios funcionales y conductuales. Así, Codina Puiggros (1994) describe en su Tratado de Neurología una serie de síndromes en los que correlaciona la sintomatología clínica con la topografía lesional. 
En general, la valoración de las personas con una disfunción cognitiva incluye, al menos, el estudio del rendimiento intelectual general, la atención, la velocidad de procesamiento de la información, la capacidad de aprendizaje y memoria, las habilidades perceptivas y motoras, el lenguaje y la comunicación, el razonamiento, la capacidad de solución de problemas y otras funciones asociadas a los lóbulos frontales [18-23].

En la evaluación neuropsicológica no se puede establecer un protocolo rígido de evaluación ni efectuar pruebas establecidas de antemano, dado que las personas conservan un conjunto de habilidades muy diversas en función de sus características personales, topografía de las lesiones, nivel de deterioro, etc. Ello exige un nivel de conocimiento de las pruebas de evaluación que permita seleccionar en cada caso las más adecuadas. La selección de los instrumentos adecuados para la evaluación específica es fundamental tanto en clínica como en la investigación.

En la interpretación de los resultados es preciso recordar que las puntuaciones tienen valor únicamente en el contexto global de toda la exploración neuropsicológica. También conviene tener en cuenta que el resultado en las pruebas de evaluación está influido no sólo por la disfunción cerebral, sino también por un conjunto de variables no neurológicas, como la edad, el nivel educativo, la profesión, la búsqueda de una posible indemnización, etc., que afectan al rendimiento. Los datos proporcionados por las pruebas de autoinforme, las escalas y los cuestionarios se deben interpretar con cautela, por la dificultad que muestran los sujetos para comprender las frases complejas y, en especial, por la reducida conciencia de las limitaciones, circunstancias que pueden afectar a la fiabilidad y a la validez de la información que proporcionan dichos instrumentos de evaluación.

De lo expuesto se deduce que los resultados de los tests y de las baterías neuropsicológicas deben considerarse como elementos complementarios, integrándose junto a otros datos, en especial con los factores de personalidad previos al traumatismo, con otras secuelas orgánicas consecuencia directa de la lesión y con las respuestas reactivas y las estrategias de afrontamiento que adoptan las personas ante los cambios que ha originado el daño cerebral.

Todo ello nos lleva a la necesidad de integrar los procedimientos de evaluación neuropsicológica y funcional para una correcta valoración de los pacientes postraumatizados craneales. Mientras que el examen neuropsicológico permite la identificación de los principales déficits, la valoración del grado funcional es esencial para conocer las incapacidades y los hándicaps.

\section{Secuelas psiquiátricas.}

La prevalencia de los trastornos neuropsiquiátricos entre los afectados por un TCE es muy elevada. Su importancia clínica y social viene determinada por varios factores:

- Suponen el principal obstáculo para obtener la reintegración social y laboral de los afectados.

- La psicopatología asociada a los TCE graves persiste e incluso puede incrementarse con el paso del tiempo.

- La sobrecarga familiar secundaria es superior a la debida a otros déficits físicos y/o cognoscitivos.

- El impacto sobre el equilibrio emocional de los familiares es alto.

En la etiopatogenia de los trastornos neuropsiquiátricos postraumáticos intervienen varios factores. Unos son dependientes del propio traumatismo (tipo, localización y gravedad del TCE); otros son previos al accidente, como la personalidad premórbida, los antecedentes neuropsiquiátricos familiares y personales, la situación psicosocial, económica y laboral en el momento del accidente, las reacciones psicológicas del paciente ante las secuelas postraumáticas y el impacto de éstas en 
la actividad laboral y en las relaciones sociales y familiares de los afectados. Esta multiplicidad de factores obliga a utilizar un modelo integrador para la exploración clínica, el diagnóstico y el tratamiento de las secuelas neuropsiquiátricas postraumáticas [24, 25].

A efectos operativos, se utilizan las clasificaciones categoriales vigentes para el diagnóstico de los trastornos neuropsiquiátricos postraumáticos.

En el DSM-IV-TR se incluyen las siguientes categorías diagnósticas en relación con los trastornos consecutivos a traumatismo craneal:

- Demencia debida a traumatismo craneal (F02.8)

- Trastorno amnésico debido a traumatismo craneal (F04)

- Trastorno cognoscitivo no especificado (F06.9): Trastorno postconmocional y Trastorno neurocognoscitivo leve.

- Cambio de personalidad debido a traumatismo craneal (F07.0)

- Trastorno psicótico debido a traumatismo craneal (F06)

- Trastorno del estado de ánimo debido a traumatismo craneal (F06.3)

- Trastornos de ansiedad debidos a traumatismo craneal (F06.4)

- Otros trastornos mentales debidos a traumatismo craneal:

- Trastorno sexual

- Trastornos del sueño

- Trastorno de la psicomotricidad (catatónico)

El Trastorno postconmocional y el Trastorno neurocognoscitivo leve están incluidos en el Apéndice B del DSM-IV-TR. Este apéndice contiene una serie de nuevas categorías y ejes diagnósticos que fueron propuestos para su posible inclusión en el DSM-V.

Los dos síndromes psiquiátricos más prevalentes, y a la vez más específicos de la psicopatología secundaria al daño cerebral adquirido (especialmente postraumático), son el Cambio de Personalidad debido a traumatismo craneal (F07.0) o el Trastorno Orgánico de la Personalidad de la ClE- 10 y el síndrome post-conmocional (F07.2), [26, 27].

Al igual que en el diagnóstico neuropsicológico, el psiquiátrico se basa en la exploración neuropsiquiátrica, mediante la entrevista con el paciente y sus allegados, complementada con técnicas de neuroimagen y eventuales tests psicológicos.

Algunos de los tests pueden ser administrados a los allegados, especialmente aquellos que exploran actividades de la vida diaria. Al paciente se le pueden administrar los mismos, lo que constituirá una gran ayuda para cuantificar la pérdida de funciones en el sujeto y/o el impacto del acontecimiento traumático sobre su vida. La comparación de ambos será útil para valorar hasta qué punto el paciente es consciente de sus limitaciones, no siendo infrecuente la ausencia de "conciencia de enfermedad". La alteración de este conocimiento, denominada "anosognosia", es un fenómeno clínico frecuentemente observado en pacientes con daño cerebral [28] y se caracteriza por la ausencia de conciencia de sus déficits neurológicos, cognoscitivos, sociales y/o emocionales.

El conocimiento que los pacientes con daño cerebral traumático tienen de sus déficits se puede situar en un continuo que admite fluctuaciones con el tiempo, de manera que con la evolución del traumatismo se produce una progresiva mayor conciencia de los déficits por parte de los pacientes, circunstancia que puede verse acompañada por un aumento del malestar psicológico y por aparición de trastornos adaptativos con síntomas emocionales mixtos.

Valoración de la repercusión funcional de las secuelas psíquicas.

Un objetivo esencial de la evaluación es determinar la capacidad del sujeto para cuidar de 
sí mismo y/o el grado de ayuda que necesita.

Para valorar la independencia funcional se han de examinar tres áreas esenciales:

I. Las habilidades del individuo para realizar diferentes actividades de la vida diaria, tanto simples (higiene personal, vestido, comida, etc.) como complejas (utilización del transporte público, ir de compras, administración de la economía, etc.)

2.El apoyo familiar y social disponible.

3. La conciencia de las limitaciones del paciente.

En la evaluación de estos aspectos, además de la información aportada por el paciente y sus allegados, en los últimos años se han desarrollado diferentes escalas que ayudan a valorar la independencia funcional de los pacientes, conocidas de modo genérico como "escalas de actividades de la vida diaria".

Las Actividades de la Vida Diaria (AVD) constituyen aquellas tareas ocupacionales que la persona lleva a cabo diariamente en función de su rol biológico, emocional, cognitivo, social y laboral. Las AVD básicas son aquellas que engloban las capacidades de autocuidado más elementales y necesarias: alimentación, aseo personal, control de esfínteres, vestido, transferencias y movilidad. Y las AVD instrumentales son aquellas que tienen un carácter más complejo, requieren de mayor elaboración para poder ser llevadas a cabo e indican la capacidad que tiene un sujeto para llevar una vida independiente en la comunidad: realización de tareas domésticas, uso del teléfono, responsabilidad en la medicación, manejo del dinero, compras, utilización de transportes públicos u otros recursos comunitarios. Algunas clasificaciones distinguen también AVD avanzadas: realización de viajes, desarrollo de aficiones, participación en grupos sociales, etc. Todas estas actividades, en realidad, pueden ser incluidas como tipos de AVD instrumentales.

Existen un gran número de escalas de valoración de actividades de la vida diaria. García Peña, Sánchez Cabeza y Miján de Castro [29] efectúan una revisión de algunas de las principales escalas de evaluación funcional con aplicación a pacientes con déficits secundarios a daño cerebral adquirido.

Entre las secuelas consecutivas a los TCE, los cambios físicos son los más evidentes; sin embargo, las alteraciones cognitivas, neurocomportamentales y emocionales constituyen el principal obstáculo para poder desarrollar actividades básicas e instrumentales de la vida cotidiana, mantener relaciones interpersonales satisfactorias y volver a una actividad laboral productiva [30]. Algunos autores defienden las medidas directas como instrumentos de evaluación, ya que ponen un énfasis especial en los aspectos ecológicos, como la autonomía del paciente y la disminución de la carga de los familiares, permitiendo la observación del comportamiento del individuo en diferentes actividades y situaciones cotidianas.

Las medidas globales de funcionamiento están formadas por escalas descriptivas que incluyen una valoración de las consecuencias psicosociales del daño cerebral y resumen varios indicadores de resultados en una puntuación única. En la evaluación de resultados globales destaca la Glasgow Outcome Scale (GOS) (Escala de resultados de Glasgow) [3I].

Esta escala se propone en la tabla VI del anexo de la Ley de Responsabilidad Civil y Seguro en la Circulación de Vehículos a Motor, en la valoración del "Deterioro de las funciones superiores cerebrales integradas, acreditado mediante pruebas específicas (Glasgow Outcome Scale)".

La Escala consta de cinco categorías o grados de discapacidad y subdivide a los pacientes en buena recuperación, moderada incapacidad, grave incapacidad, estado vegetativo persistente y muerte. 
Grado 1: Buena recuperación. Se reintegra a su ocupación previa. Implica el regreso a la vida normal, a pesar de que puedan quedar déficits neurológicos y psicológicos mínimos. El regreso al trabajo no es un índice real de recuperación, puesto que depende de circunstancias particulares. También deben tenerse en cuenta otros aspectos, como las relaciones sociales, actividades recreativas y relaciones familiares.

Grado 2: Moderada incapacidad. Pueden hacer sus tareas diarias, pero no reintegrarse al trabajo o a los estudios (discapacitados pero independientes). Son independientes en cuanto a su vida diaria, pero algunas actividades previas no las pueden realizar por los déficits físicos, neuropsicológicos y/o neuropsiquiátricos. Estas secuelas pueden ocasionar una disrupción muy marcada del contexto familiar y social del paciente.

Grado 3: Grave incapacidad. Necesitan asistencia para sus tareas diarias, pero no cuidados institucionales (conscientes pero discapacitados). Pacientes que requieren ayuda en actividades de la vida diaria por razones de incapacidad mental o física o ambas. Puede variar desde una dependencia continua total (alimentación y lavado) hasta necesitar ayuda en una sola actividad, como vestirse, moverse por casa o salir a comprar.

Grado 4: Estado vegetativo persistente. Pacientes sin respuestas significativas a estímulos ambientales. Son incapaces de obedecer órdenes simples o decir palabras. Los pacientes vegetativos respiran espontáneamente, pueden abrir los ojos y seguir algún objeto en movimiento, deglutir comida depositada en su boca, mostrar respuestas reflejas a estímulos dolorosos y posturales y tener ciclos de sueño y vigilia normales.

Grado 5: Muerte.

Por último, la Ley 39/2006, de 14 de diciembre, de Promoción de la Autonomía Personal y Atención a las personas en situación de dependencia [32] tiene por objeto regular las condiciones básicas que garanticen la igualdad en el ejercicio del derecho subjetivo a la promoción de la autonomía personal y atención a las personas en situación de dependencia, en los términos establecidos en las leyes, mediante la creación de un Sistema para la Autonomía y Atención a la Dependencia, con la colaboración y participación de todas las Administraciones Públicas y la garantía por la Administración General del Estado de un contenido mínimo común de derechos para todos los ciudadanos en cualquier parte del territorio del Estado español.

En el Real Decreto 504/2007, de 20 de abril [33], se aprueba el baremo de valoración de la situación de dependencia establecido por la Ley 39/2006, de I 4 de diciembre, de Promoción de la Autonomía Personal y Atención a las personas en situación de dependencia.

El baremo valora la capacidad de la persona para llevar a cabo las actividades básicas de la vida diaria, así como la necesidad de apoyo y supervisión para su realización por personas con discapacidad intelectual o con enfermedad mental (Art. 27.4 de la Ley), así como en aquellas otras situaciones en que las personas puedan tener afectada su capacidad perceptivocognitiva.

En la Guía elaborada, incluida en el Apéndice del presente artículo, se proponen una serie de pruebas para efectuar la valoración funcional de las secuelas consecutivas a TCE.

\section{LIMITACIONES EN LA EVALUACIÓN CLÍNICO-FORENSE DEL DAÑO PSíQUICO CONSECUTIVO A LOS TRAUMATISMOS CRANEOENCEFÁLICOS:}

Son varias las limitaciones existentes en la evaluación de las secuelas neuropsicológicas y neuropsiquiátricas derivadas de un traumatismo craneoencefálico. Así, el Médico Forense a menudo no cuenta con los datos suficientes para efectuar una correcta evaluación. 
I. Es difícil determinar la presencia o ausencia de un estado patológico anterior.

2. En el daño psíquico existe dificultad al aplicar la relación de causalidad (criterios cronológico, cuantitativo, topográfico, de continuidad sintomática y de exclusión).

3. La naturaleza subjetiva de la información clínica aportada dificulta el diagnóstico y facilita la simulación, circunstancias que complican la peritación.

4. Entre las secuelas neuropsicológicas de los traumatismos craneoencefálicos es frecuente la falta de conciencia de los déficits, lo cual puede dar lugar a una infravaloración de la magnitud de las secuelas. El error pericial puede consistir en pasar por alto un deterioro cognitivo marcado, especialmente si no se dispone de una evaluación neuropsicológica de calidad.

Uno de los principales problemas que surgen en la evaluación pericial de estas secuelas en los traumatizados craneales radica en que la aplicación de la mayoría de las baterías neuropsicológicas es compleja para el médico forense encargado de la valoración pericial del sujeto.

Para paliar algunas de éstas limitaciones se propone para el futuro la intervención de un neuropsicólogo forense en las peritaciones de secuelas neuropsicológicas consecutivas a traumatismos craneoencefálicos. Por otro lado, mediante los test neuropsicológicos dispondremos de información útil para llevar a cabo una investigación.

Conviene despejar en futuras investigaciones algunas incógnitas como, por ejemplo, la valoración del impacto funcional de las secuelas neuropsicológicas y neuropsiquiátricas consecutivas a los traumatismos craneoencefálicos mediante escalas funcionales.

\section{GUÍA \\ PARA LA EVALUACIÓN DE SECUELAS NEUROPSICOLÓGICAS Y NEUROPSIQUIÁTRICAS DE LOS TRAUMATISMOS CRANEOENCEFÁLICOS}

No EXP. CMF:

FECHA:

JUZGADO No

CAUSA No

FORENSE:

1. DATOS DEL EXPLORADO

PRIMER APELLIDO:

NOMBRE:

EDAD:

SEGUNDO APELLIDO:

ESTADO CIVIL:

FECHA DE NACIMIENTO:

NIVEL EDUCATIVO:

PROFESIÓN:

STATUS LABORAL PREVIO AL TCE:

LUGAR DE RESIDENCIA:

DOMICILIO:

TELÉFONO: 
2.- FECHA DEL TRAUMATISMO CRANEOENCEFÁLICO

DIA: MES: AÑO: HORA:

DIA SEMANA:

3.- ETIOLOGÍA MÉDICO-LEGAL DEL TCE

3.1. ACCIDENTE DE TRÁFICO

3.2. AGRESIÓN

3.3. ACCIDENTE LABORAL

3.4. ACCIDENTE CASUAL

4.- ANTECEDENTES PERSONALES

4.1. ANTECEDENTES PERSONALES :

4.1.1. SOMÁTICOS.

4.1.2. Psíquicos.

4.1.3. Tóxicos.

4.2. ACCIDENTES PREVIOS

5.- SEVERIDAD DEL TRAUMATISMO CRANEOENCEFÁLICO

5.1. ESCALA DE COMA DE GLASGOW

5.2. DURACIÓN DEL COMA

5.3. AMNESIA POSTRAUMÁTICA

6.- NEUROIMAGEN

6.1. TAC CEREBRAL EN LAS PRIMERAS 48 HORAS

6.2. TAC O RMN CEREBRAL DESPUÉS DE LOS 3 MESES

7.- DIAGNOSTICO DE LESIONES Y COMPLICACIONES INICIALES

7.1. LESIONES EN CARA Y CUERO CABELLUDO

7.2. FRACTURA CRANEAL

7.3. HEMORRAGIA INTRACRANEAL

7.4. LATERALIDAD DEL DAÑO CEREBRAL

8.- COMPLICACIONES EVOLUTIVAS

8.1. CRISIS EPILÉPTICAS

8.2. HIDROCEFALIA

8.3. INFECCIONES GRAVES

8.4. OTRAS

9.- TRATAMIENTO

9.1. FASE AGUDA.

- Estancia EN UCl y NeUROCIRUgía

- INTERVENCIÓN NEUROQUIRÚRGICA

9.2. REHABILITACIÓN EN RÉGIMEN DE HOSPITALIZACIÓN

- DESCRIBIR TIPOS DE REHABILITACIÓN

9.3. REHABILITACIÓN AMBULATORIA

- DESCRIBIR TIPOS DE REHABILITACIÓN 


\section{0.- VALORACIÓN DEL DAÑO CORPORAL Y PSIQUICO}

10.1. DETERMINACIÓN DE LOS TIEMPOS LEGALES:

10.1.1. DURACIÓN DEL INGRESO HOSPITALARIO (EN DÍAS)

10.1.2. DURACIÓN DEL TIEMPO INVERTIDO EN LA CURACIÓN O ESTABILIZACIÓN LESIONAL (EN DÍAS)

10.1.3. DURACIÓN DEL TIEMPO QUE HA PERMANECIDO IMPEDIDO PARA SUS OCUPACIONES HABITUALES (EN DÍAS)

\section{1.- SECUELAS}

11.1. NEUROLÓGICAS (epilepsia)

11.2. MOTORAS Y SENSITIVAS (hemiplejia, ataxia, hemiparexia, etc)

11.3. SENSORIALES

11.4. SECUELAS NEUROPSICOLÓGICAS. Valoración clínica y pruebas válidas para su valoración.

11.4.1. CONCIENCIA

- Valoración Clínica: Coma, estupor, estado crespuscular, obnuvilación, hipervigilante, etc.

- Prueba: Escala de coma de Glasgow

\subsubsection{ORIENTACIÓN}

- Valoración ClínICa:

¿Conoce sus datos personales?:

¿Cómo se llama?

¿Qué edad tiene?

¿A que se dedica (profesión)?

¿Cuál es su estado civil?

¿Dónde vive?

¿Cuál es su número de teléfono?

¿Sabe el día en que vive?:

¿En que año estamos?

¿En que estación o época del año estamos?

¿En que mes estamos?

¿Qué día de la semana es hoy?

¿Qué día del mes es hoy?

¿Sabe donde está?

¿En que país estamos?

¿En que provincia estamos?

¿En que ciudad estamos?

¿Dónde estamos ahora?

¿En que piso estamos?

- PrUEBA: Registro de Orientación Subtest de Barcelona

\subsubsection{ATENCIÓN}

- VAlORACIÓN CLÍNICA: Observación directa de su funcionamiento durante la exploración (necesidad de instigación, fatigabilidad, enlentecimiento, etc.).

¿Es capaz de mantener la atención en una tarea?

¿Tarda mas tiempo que antes de la lesión?

¿Se cansa?

¿Puede hacer dos cosas a la vez? 
- PRUEBAS:

- Digitos y clave de números (subprueba WAIS)

- STROOP: Test de Colores y Palabras. Para valorar atención selectiva

- Subtest de Atención Visuográfica de Barcelona

- Trail Making Test (formas A y B). Para atención alternante

- PASAT. Para valorar atención dividida

\subsubsection{MEMORIA}

- Valoración Clínica: Preguntas que hagan referencia a la memoria prospectiva, retrospectiva y autobiográfica:

¿Reconoce a las personas de su entorno familiar?

¿Conoce la relación que mantiene con las personas de su entorno? ¿Recuerda los hechos mas relevantes de su vida hasta el momento de la lesión?

¿Recuerda los hechos más relevantes de su vida desde el momento de la lesión?

¿Recuerda lo que hizo ayer?

¿Recuerda los recados que se le dan?

- PRUEBAS:

- Escala de Memoria de Wechler-Revisada: (WMS-R)

- Letras y números (Subpruebas del WAIS)

- Subtest de información del WAIS

- Test de Rivermead de memoria conductual

- Memoria de Figura compleja de Rey-Osterrieth

- TAVEC

- Examen de la memoria BNS

11.4.5. LENGUAJE Y COMUNICACIÓN

- Valoración Clí́nICA:

¿Dificultades para la denominación de objetos de uso común? ¿Disminución y pobreza de la fluidez verbal?

¿Dificultad para expresar las ideas de modo claro y conciso?

¿Dificultades de comprensión de instrucciones complejas?

¿Aumento del empleo de circunloquios y parafasias semánticas?

¿Pide ayuda cuando lo necesita?

¿Mantiene conversaciones?

¿Escribe (en papel, tablero u ordenador?

¿Comprende lo que se le dice?

¿Comprende lo que lee?

¿Utiliza el teléfono?

- PRUEBAS:

- Test de denominación de Boston

- Mini-Mental (Subtest lenguaje)

- Diagnóstico de la Afasia Puebla/Sevilla

11.4.6. Pensamiento, RaZONAMIENTO Y FUnCIONES EJeCutivas

- VAlORACIÓN CLÍNICA:

¿Tiene dificultades para secuenciar actividades?

¿Maneja aparatos como el móvil, la TV, etc.? 
- PRUEBAS:

- Clasificación de cartas de Wisconsin

- Trail Making Test (forma B)

11.4.7. PERCEPCIÓN Y CAPACIDADES VISUOPERCEPTIVA Y VISUOCONSTRUCTIVA

- Valoración CLÍNICA:

¿Reconoce objetos, fotos...?

¿Describe las características de objetos presentados visualmente (forma, color)?

¿Empareja objetos (series de fotografías u objetos)?

¿Copia dibujos lineales o simétricos (flor, casa, bicicleta)?

¿Identifica visualmente (reconocimiento de los atributos de un estímulo visual)?

¿Conoce objetos no presentados visualmente (características)?

¿Reconoce objetos por el tacto (con los ojos cerrados)?

¿Utiliza correctamente diferentes utensilios?

¿Omite alguna parte de su campo visual?

- PRUEBAS:

- Figura compleja de Rey

- Rompecabezas y cubos (Subtest WAIS)

11.4.8. CONDUCTA Y ESTADO EMOCIONAL

- VAlORACIÓN CLÍnICA: Preguntar al lesionado y a la familia acerca de cambios de personalidad: impulsividad, egocentrismo, infantilismo, irritabilidad, escasa tolerancia a la frustración, desinhibición social, desinhibición sexual, suspicacia, rigidez de conducta, oposicionismo, heteroagresividad verbal y/o física, autoagresividad, aislamiento social, apatía, perseveración, conducta manipulativa, apatía, depresión y euforia.

- PRUEBAS:

- La versión española de la Neurobehavioral Rating Scale (NRS).

11. 5. DIAGNÓSTICO PSIQUIÁTRICO

El diagnóstico psiquiátrico se realiza según los criterios diagnósticos del DSM-IV-TR para cada trastorno debido a traumatismo craneal:

11.5.1. (F02.8) DEMENCIA DEBIDA A TRAUMATISMO CRANEAL (294. 1x)

11.5.2. (F04) TRASTORNO AMNÉSICO DEBIDO A TRAUMATISMO CRANEAL (294.0)

11.5.3. (F06. 9) TRASTORNO COGNITIVO NO ESPECIFICADO (294.9)

11.5.3.1. Trastorno neurocognitivo leve

11.5.3.2. Trastorno posconmocional

11.5.4. (FO7.0) CAMBIO DE PERSONALIDAD DEBIDO A TRAUMATISMO CRANEAL (310.1)

11.5.5. (FO6.X) TRASTORNO PSICÓTICO DEBIDO A TRAUMATISMO CRANEAL (293.XX)

11.5.6. (FO6.xx) TRASTORNO DEL ESTADO DE ÁNIMO DEBIDO A TRAUMATISMO CRANEAL (293.83)

11.5.7. (FO6.4) TRASTORNO DE ANSIEDAD DEBIDO A TRAUMATISMO CRANEAL (293.84) 
11.5.8. OTROS TRASTORNOS MENTALES DEBIDOS A TRAUMATISMO CRANEOENCEFÁLICO:

- Trastorno sexual

- Trastornos del sueño

- Trastornos de la psicomotricidad (Catatónico)

- Otros.

\section{VALORACIÓN FUNCIONAL}

12.1. DISPONIBILIDAD PARA EL TRABAJO: EMPLEO

Capacitado

Parcialmente incapacitado

Totalmente incapacitado

12.2. VIDA EN LA COMUNIDAD

Capacitado

Parcialmente incapacitado

Totalmente incapacitado

12.3. MANEJO DE DINERO

Independiente

Precisa supervisión

Totalmente dependiente

12.4. DESPLAZAMIENTOS

Independiente

Dependiente en uso de transporte público

Dependiente para trayectos cortos

Totalmente dependiente

12.5. PREPARACIÓN DE COMIDAS

Independiente

Precisa supervisión

Precisa de ayuda física parcial

Totalmente dependiente

12.6. ASEO PERSONAL

Independiente

Precisa supervisión

Precisa de ayuda física parcial

Totalmente dependiente

12.7. VESTIDO

Independiente

Precisa supervisión

Precisa de ayuda física parcial

Totalmente dependiente

12.8. ALIMENTACIÓN

Independiente

Precisa supervisión

Precisa de ayuda física parcial

Totalmente dependiente 


\section{9. CONTROL DE ESFÍNTERES}

Independiente

Precisa control externo

Incontinencia urinaria

Doble incontinencia

- PRUEBAS:

- Glasgow Outcome Scale- GOS: Escala de resultados de Glasgow.

- Escala de evaluación de la actividad global (EEAG): DSM-IV-TR.

- Escala de valoración de la discapacidad (DRS).

- Medida de Independencia Funcional (FIM).

- Medida de Evaluación Funcional (FAM).

- Baremo de valoración de la situación de dependencia establecido en la Ley 39/2006 y aprobado en el Real Decreto 504/2007.

- Inventario de destrezas adaptativas (CALS).

- Clasificación Internacional del Funcionamiento, de la

Discapacidad y de la Salud (CIF).

\section{BIBLIOGRAFÍA:}

I. Aso J. Valoración de las lesiones causadas a las personas en accidentes de circulación a partir de la Ley 34/03. Barcelona. ElsevierMasson, 2006.

2. Muñoz Céspedes, J.M., Paúl-Lapedriza, N., Pelegrin-Valero, C. y Tirapu-Ustarroz, J. Factores pronóstico en los traumatismos craneoencefálicos. Rev Neurol 2001;32:351-364.

3. León Carrión, J., y Domínguez Morales, R. Valoración de las secuelas mentales y psiquiátricas derivadas del daño cerebral traumático: cuando, que y como. Rev Neuropsicologia 2005; 7 : 35-49.

4. Moreno, P. Epidemiología, clasificación y pronóstico de los traumatismos craneoencefálicos. Infor Psiquiátricas 1999;158:287-302. 5. Bruns, J., Hauser, W. A. The Epidemiology of Traumatic Brain Injury. Rev Epilepsi 2003; 10: 2-10.

6. Ley 34/2003, de 4 de noviembre, de modificación y adaptación a la normativa comunitaria de la legislación de seguros privados. BOE núm 265, de 5 de noviembre de 2003.

7. Asociación Psiquiátrica Americana. DSM-IV-TR. Manual de Diagnóstico y Estadístico de los Trastornos Mentales. Barcelona: Masson, 2000.

8. Organización Mundial de la Salud: CIE-10. Trastornos mentales y del comportamiento. Criterios diagnósticos de investigación. Madrid: Méditor, 1994.

9. Pelegrin, C., Muñoz Céspedes, J. M. y Quemada, J. I. Neuropsiquiatría del daño cerebral traumático: Aspectos clínicos y terapéuticos. Barcelona: Prous Science, 1997.

10. Codina Puiggros, A. Tratado de Neurología. Síndromes topográficos cerebrales. Madrid: ELA, 1994.

II. Urigüen Saiz, M. Traumatismos craneoencefálicos. Revisión fisiopatológica, clasificación y presentación clínica. Medicine 1998;7:4200-4208.
12. Gisbert Calabuig, J.A. Medicina Legal y Toxicología. Barcelona: Masson, 2004.

I3. Quemada, J.I., Hormaechea, J.A. y Muñoz Céspedes, J.M. La peritación psiquiátrica y neuropsicológica del daño cerebral traumático y la Ley 30/95. Actas Esp Psiquiatr 2003;31:353360.

14. Ministerio de Hacienda, Resolución de 17 de enero de 2008, de la Dirección General de Seguros y Fondos de Pensiones, por la que se da publicidad a las cuantías de las indemnizaciones por muerte, lesiones permanentes e incapacidad temporal, que resultarán de aplicar durante 2008, el sistema para valoración de los daños y perjuicios causados a las personas en accidentes de circulación. BOE núm 2I, de 24 de enero de 2008.

15. Borobia C. Valoración del Daño Corporal: Legislación, metodología y prueba pericial médica. Barcelona: Elsevier-Masson, 2006.

16. Pérez Pineda B, García Blázquez M. Valoración y diagnóstico de las secuelas en los traumatismos craneoencefálicos. Manual de valoración y baremación del daño corporal ( $10^{a}$ edición). Granada: Comares, 2000.

17. Ley 34/2003, de 4 de noviembre, de modificación y adaptación a la normativa comunitaria de la legislación de seguros privados. BOE $n^{0} 265$, de 5 de noviembre de 2003.

18. Benedet, M. J. Evaluación neuropsicológica. Bilbao: Desclée de Brouwer, 1987.

19. Boget, T., Cañizares, S. y Penadés, R. Avances en evaluación y rehabilitación neuropsicológica. En M. Lameiras y J. M. Faílde (eds.). La psicología clínica y de la salud en el siglo XXI. Posibilidades y retos. Madrid: Dykinson, 2000.

20. Christensen, L., Manga, D. y Ramos, F. LURIA_DN. Diagnóstico neuropsicológico de adultos. Madrid: Tea, 2002. 
21. Ariza González, M., Pueyo Benito, R. y Serra Grabulosa JM. Secuelas neuropsicológicas de los traumatismos craneoencefálicos. Anales Psicol 2004:2:303-316.

22. Junqué, C., Bruna, 0. y Mataró, M. Traumatismos craneoencefálicos. Un enfoque desde la neuropsicología y la logopedia. Barcelona: Masson, 1998.

23. Junqué, C. Secuelas neuropsicológicas de los traumatismos craneoencefálicos. Rev Neuropsicol 1999;28:423-430.

24. Quemada, J.I., Hormaechea, J.A. y Muñoz Céspedes, J.M. La peritación psiquiátrica y neuropsicológica del daño cerebral traumático y la Ley 30/95. Actas Esp Psiquiatr 2003;31:353-360.

25. Quemada, J.I. Valoración neuropsiquiátrica y cognitiva. Valoración y tratamiento del enfermo mental. Madrid: Aran, 2002. 26. Quemada, J.I., Sánchez Cubillo, I. y Muñoz Céspedes J.M. El Trastorno Orgánico de la Personalidad: análisis conceptual y estrategias para la investigación. Actas Esp Psiquiatr 2007;35:I15-I2I. 27. Francisco Maiz, M. L. Tesis Doctoral: Aportaciones a la evaluación clínico-forense de secuelas neuropsiquiátricas y neuropsicológicas de los traumatismos craneoencefálicos. UPV-EHU, 2007. 28. Urrutikoetxea, I., Muñoz Céspedes, J.M., García, M. y Iruarrizaga,
I. La conciencia de los déficits en la rehabilitación de las personas con un daño cerebral. Ansiedad y Estrés 1999;15:313-325.

29. García Peña, M., Sánchez Cabeza, A. y Miján de Castro, E. Evaluación funcional y terapia ocupacional en el daño cerebral adquirido. Rehabilitación 2002;36:167-175.

30. Zabala Rabadan, A., Muñoz Céspedes, J.M. y Quemada Ubis, J.I. Efectividad de la rehabilitación neuropsicológica en pacientes con daño cerebral adquirido: fundamentos y dificultades metodológicas en la investigación. Rehabilitación 2003;37:103-II2.

3I. Wilson, J., Pettigrew, L. y Teasdale, G.M. Structured Interviews for the Glasgow Outcome Scale and the Extended Glasgow Outcome Scale: Guidelines for Their Use. J Neurotrauma 1998;15:573-585. 32. Ley 39/2006, de 14 de diciembre, de Promoción de la Autonomía Personal y Atención a las personas en situación de dependencia.

33. Real Decreto 504/2007, de 20 de abril, en el que se aprueba el baremo de valoración de la situación de dependencia establecido por la Ley 39/2006, de 14 de diciembre, de Promoción de la Autonomía Personal y Atención a las personas en situación de dependencia. 